\title{
Effect of Ball Scribing on Relative Permeability of Grain-oriented Electrical Steel
}

\author{
Hao WANG ${ }^{1,2)}$, Changsheng $L^{1) \dagger}$, Tao ZHU ${ }^{1)}$, Nkwachukwu Chukwuchekwa ${ }^{2)}$, Ban $C A I^{1)}$ \\ and Gang $\mathrm{HUO}^{1,3)}$ \\ 1) State Key Laboratory of Rolling and Automation, Northeastern University, Shenyang 110819, China \\ 2) Wolfson Centre for Magnetics, Cardiff University, Cardiff CF24 3AA, UK \\ 3) Pohang Cold Strip Rolling Co. Ltd of Bensteel, Benxi 117021, China \\ [Manuscript received 15 January 2013, in revised form 3 May 2013] \\ (C) The Chinese Society for Metals and Springer-Verlag Berlin Heidelberg
}

\begin{abstract}
Effect of ball scribing on relative permeability of conventional grain-oriented (CGO) and high permeability grain-oriented (HGO) electrical steel was investigated. The samples were scribed with spacing of $2 \mathrm{~mm}, 4 \mathrm{~mm}$, $8 \mathrm{~mm}$ and $16 \mathrm{~mm}$. The results show that after ball scribing with $16 \mathrm{~mm}$ width at $1.0 \mathrm{~T}$, relative permeability of both CGO and HGO steels was increased by $109 \%$ and $80 \%$, respectively. Relative permeability rises as the scribing space increases, with the movement of the peak value of relative permeability to a higher flux density. Relational models describing relative permeability and flux density were constructed with high accuracy based on experimental data. The experimental data curves were analyzed during the magnetizing process.
\end{abstract}

\section{KEY WORDS: Ball scribing; Grain-oriented electrical steel; Relative permeability; Rela- tional modeling}

\section{Introduction}

Grain-oriented electrical steel has a wide application as core material in various transformers, amplifiers, electromagnetic switches and voltage regulators $^{[1]}$. Excellent magnetic properties make grain-oriented electrical steel suitable material for iron core of directional magnetic field such that the magnetic induction is increased and hysteresis loss is reduced $^{[2,3]}$. Currently, grain-oriented electrical steel becomes one of the most attractive and potential materials for large-scale fossil-fired, thermal power and nuclear plants due to its excellent combination of weight-lightening and energy saving ${ }^{[4,5]}$.

Relative permeability received considerable attention owing to the increasing demand for magnetic shielding, such as application in magnetic resonance imaging (MRI) scans and magnetically levitated trains ${ }^{[6]}$. As a significant magnetic property, relative permeability is closely related not only to

\footnotetext{
† Corresponding author. Prof., Ph.D.; Tel.: $\quad+8624$ 83687749; Fax: +86 24 23906472; E-mail address: lics@ral.neu.edu.cn(Changsheng LI)
}

DOI: $10.1007 / \mathrm{s} 40195-013-0021-3$ alloy elements, grain size, microstructure defects, but also to external factors including strain and stress, magnetizing field and temperature. Research efforts for obtaining high permeability have been in $\operatorname{progress}^{[7-9]}$.

European Electrical Steels developed a ball scribing technique to address the problem of re-coating ${ }^{[10]}$. In this method, a steel ball is pressed onto the steel surface, and with the appropriate pressure a region of permanent plastic damage occurs. This method is not anneal proof, but it does not affect the steel coating so recoating treatment could be avoided, and it is appropriate for transformer core plate which is routinely fitted without re-anneal. Some researches have been carried out concerning magnetic properties after scribing ${ }^{[11,12]}$. Compared with other mechanical scribing methods, ball scribing would not damage insulation layers on the electrical steel surface, therefore, the problems of low lamination factor, high magnetostriction and noise caused by other scribing methods could be effectively avoided ${ }^{[13,14]}$. In this paper, ball scribing is used to investigate its effect on relative permeability. On the basis of experimental data, models for relative permeability and flux density were established. Advantages of ball scribing to electrical 
Table 1 Chemical composition of the experimental steels C711 and H668 (wt.\%)

\begin{tabular}{ccccccccc}
\hline Steel & $\mathrm{Si}$ & $\mathrm{C}$ & $\mathrm{Mn}$ & $\mathrm{S}$ & $\mathrm{Cu}$ & $\mathrm{P}$ & $\mathrm{Al}$ & $\mathrm{Fe}$ \\
\hline $\mathrm{C} 711$ & 3.05 & 0.032 & 0.065 & 0.020 & 0.028 & 0.012 & 0.008 & Bal. \\
$\mathrm{H} 668$ & 3.09 & 0.054 & 0.072 & 0.018 & 0.075 & 0.015 & 0.010 & Bal. \\
\hline
\end{tabular}

Table 2 Initial magnetic properties of $\mathrm{C} 711$ and H668 steels at $1.0 \mathrm{~T}$

\begin{tabular}{ccccc}
\hline Steel & Iron loss $(\mathrm{W} / \mathrm{kg})$ & Relative permeability & Coercive force $(\mathrm{A} / \mathrm{m})$ & MBNrms $(\mathrm{mV})$ \\
\hline $\mathrm{C} 711$ & 0.711 & 799.4 & 282.9 & 0.391 \\
$\mathrm{H} 668$ & 0.668 & 2473.7 & 287.6 & 0.447 \\
\hline
\end{tabular}

Note: MBNrms - root mean square of magnetic Barkhausen noise

steel used for magnetic shielding were also analyzed.

\section{Experimental}

The grain-oriented electrical steel samples included conventional grain-oriented (CGO) electrical steel sheets and high permeability grain-oriented (HGO) electrical steel sheets. Two types of electrical steel were named as $\mathrm{C} 711$ and $\mathrm{H} 668$ respectively, and their chemical composition are shown in Table 1.

The samples were prepared as standard Epstein samples with size of $350 \mathrm{~mm} \times 30 \mathrm{~mm} \times 0.3 \mathrm{~mm}$. Initial magnetic properties of $\mathrm{C} 711$ and $\mathrm{H} 668$ are shown in Table 2. Ball scribing was carried out in selfdesigned ball scribing instrument where scribing spacing of $2 \mathrm{~mm}, 4 \mathrm{~mm}, 8 \mathrm{~mm}$ and $16 \mathrm{~mm}$ was achieved. The scribing direction is perpendicular to the rolling direction.

Measurements of relative permeability were made in an Epstein frame, using a data acquisition system. Each measurement group is composed of 3 samples, and each sample was measured 3 times for an average value. Due to various influencing factors, each sample was demagnetized along the rolling direction before each measurement to obtain accurate experimental data. Magnetizing yokes, coils and samples were placed in a noise shielding room in order to reduce the effect of environmental noise. Temperature was controlled at $25{ }^{\circ} \mathrm{C}$ with an air conditioner to eliminate temperature disturbance. Computers for collecting data were placed in another room to avoid interference.

\section{Results and Discussion}

Relative permeabilities of C711 before and after ball scribing are shown in Fig. 1. It is obvious that after scribing relative permeability first rises and then falls, and as the scribing spacing is larger the corresponding relative permeability is growing substantially. Permeabilities are increased by $42 \%$ and $109 \%$ with scribing spacings of $2 \mathrm{~mm}$ and $16 \mathrm{~mm}$ at $1.0 \mathrm{~T}$. Increase of scribing spacing also makes peak points of relative permeability move right, which indicates that CGO electrical steel arrives peak relative permeability points at higher flux densities. At lower flux densities, relative permeability of C711 with different scribing spacing is almost the same, which differs from

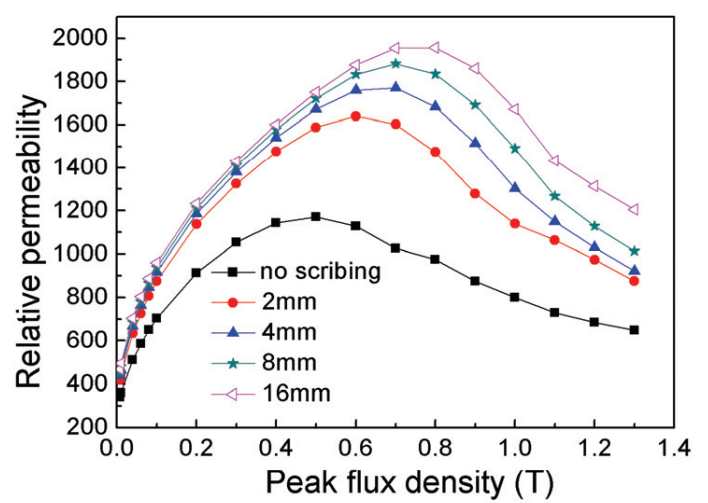

Fig. 1 Comparison of relative permeability variations of C711 before and after ball scribing for different spacing

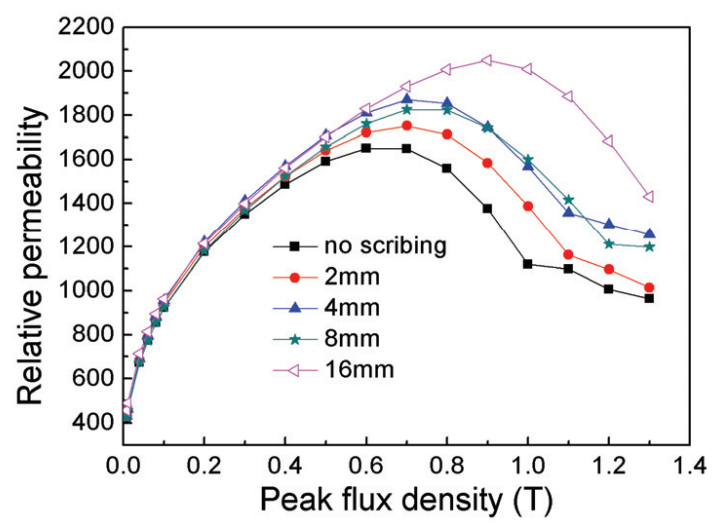

Fig. 2 Comparison of relative permeability variations of H668 before and after ball scribing for different spacing

the fact that remarkable difference appears at higher flux densities without scribing.

As shown in Fig. 2, relative permeabilities of H668 before and after ball scribing are compared. Relative permeability of H668 also shows a similar trend as that of $\mathrm{C} 711$ which first ascends and then descends. Except that relative permeabilities of HGO electrical steel with scribing width of $4 \mathrm{~mm}$ and $8 \mathrm{~mm}$ are roughly equal, other relative permeabilities ascend as the flux density rises. Relative permeabilities are increased by $24 \%$ and $80 \%$ with scribing width of $2 \mathrm{~mm}$ and $16 \mathrm{~mm}$ at $1.0 \mathrm{~T}$. Increase of scribing spacing also makes HGO electrical steel hit peak relative permeability points at higher flux densities. At lower flux 
densities, relative permeability of C711 does not show obvious variation for samples with different scribing spacing, which differs from the fact that a notable gap appears at higher flux densities without scribing. At low flux densities, there exists little difference between the relative permeabilities of $\mathrm{C} 711$ with and without scribing space, while substantial difference appears at higher flux densities.

Transverse and longitudinal relative permeabilities of electrical steel vary greatly, which is mainly due to strong crystal magnetic anisotropy, and while the external magnetic field does not magnetize uniformly along certain magnetization direction a preferred orientation distribution of the magnetic lines formed. The direction of a large magnetic relative permeability will first fill the magnetic field lines, for the tested material which is rolling direction in this paper. While the magnetization along rolling direction arrives at its maximum relative permeability, filling of magnetic field lines achieves saturated status where shielding energy also hits its maximum. As the external magnetic field continuously increases the magnetic lines fills along other directions, and relative permeability along the rolling direction begins to decay. Relative permeability along other directions successively reaches maximum, however, relative permeability along these directions is smaller than that along rolling direction. Although there is an increase of relative permeability along other directions, relative permeability along rolling direction gradually decays. Therefore, magnetic shielding effectiveness will not decay rapidly but descends steadily after inflexion points appear.

Further comparison of effect on relative permeabilities of $\mathrm{C} 711$ and H668 is shown in Fig. 3, where the relative permeabilities before scribing and average value of relative permeabilities after scribing were compared. Apparent variation occurs after scribing. Before scribing, relative permeability of HGO electrical steel is substantially higher than that of CGO electrical steel, and both increase at low flux densities and then decrease as the flux density rises. CGO electrical steel arrives at peak relative permeability of 1169.9 at $0.5 \mathrm{~T}$, while HGO electrical steel amounts peak relative permeability of 1648.7 at $0.7 \mathrm{~T}$. Marked difference could be seen before scribing, especially at $0.6 \mathrm{~T}$ a permeability gap of 421.5 appears; while after scribing the variation curves could be separated into two sections, as shown in Fig. 3(b), and peak relative permeability acts as the cut-off point. In the first section, there is no apparent difference, and in the second section both curves decrease with identical relative permeability difference. Their relative permeability difference is 42 at $1.0 \mathrm{~T}$, which is approximately $1 / 10$ of relative permeability before scribing.

Relation models between relative permeability and flux density were built. It can be seen from Fig. 3 that after scribing with different spacing scribing, the relative permeability variations are similar. Average
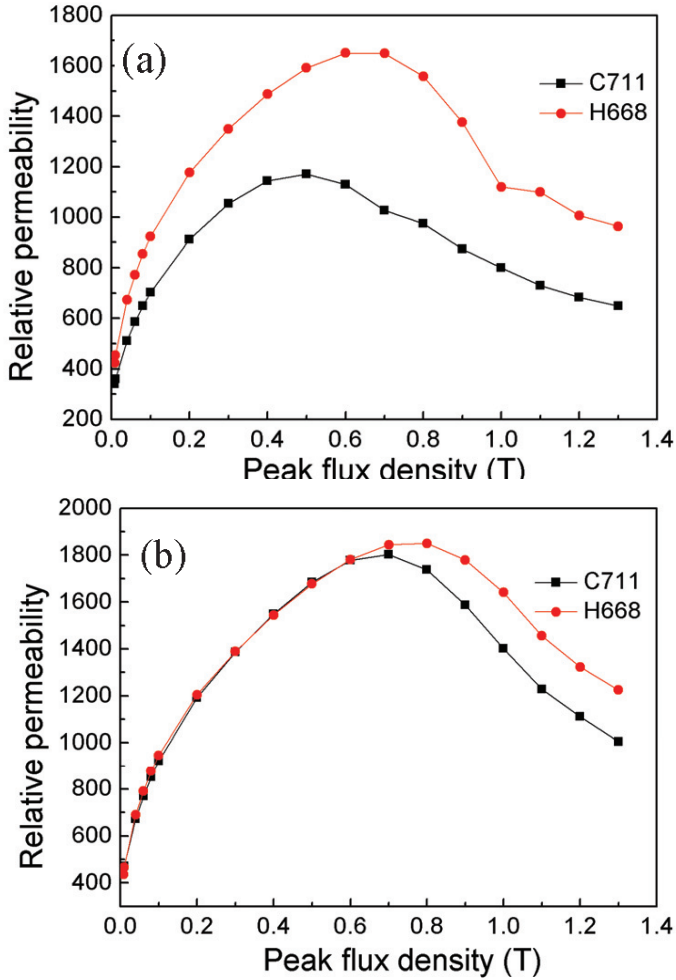

Fig. 3 Comparison of relative permeability variation of C711 and H668 before (a) and after (b) ball scribing

value of relative permeability were used in the modelling, and variation curves could be divided into four zones, the first two and the fourth zones are linear zones, while the third zone is a nonlinear zone. Modelling equations were built as follows.

The first and second linear zones are identical for both CGO and HGO electrical steel, as shown in the following equation:

$$
\begin{aligned}
& \mu=5246.4 B+427.7(\text { when } 0.008 \mathrm{~T} \leq B \leq 0.1 \mathrm{~T}) \\
& \mu=1879.6 B+783.0(\text { when } 0.1 \mathrm{~T} \leq B \leq 0.5 \mathrm{~T})
\end{aligned}
$$

where $\mu$ is the relative permeability, $B$ is the magnetic density.

For CGO electrical steel, model for the third and fourth zone is established as:

$$
\begin{aligned}
& \mu=-4129.9(B-0.7)^{2}+1800.1(\text { when } 0.5 \mathrm{~T} \leq B \leq 0.9 \mathrm{~T}) \\
& \mu=-1456.4 B+2868.4(\text { when } 0.9 \mathrm{~T} \leq B \leq 1.3 \mathrm{~T})
\end{aligned}
$$

Similarly, for HGO electrical steel, model for the third and fourth zone is established as:

$$
\begin{aligned}
& \mu=-3116.3(B-0.75)^{2}+1853.1(\text { when } 0.5 \mathrm{~T} \leq B \leq 0.9 \mathrm{~T}) \\
& \mu=-1381.1 B+2999.3(\text { when } 0.9 \mathrm{~T} \leq B \leq 1.3 \mathrm{~T})
\end{aligned}
$$

Modelling construction of H668 electrical steel is shown in Fig. 4, after calculation and analysis fitting degrees of both linear parts and nonlinear part are quite high, and linear fitting degree is above 0.99 and for nonlinear part it is above 0.97 . 

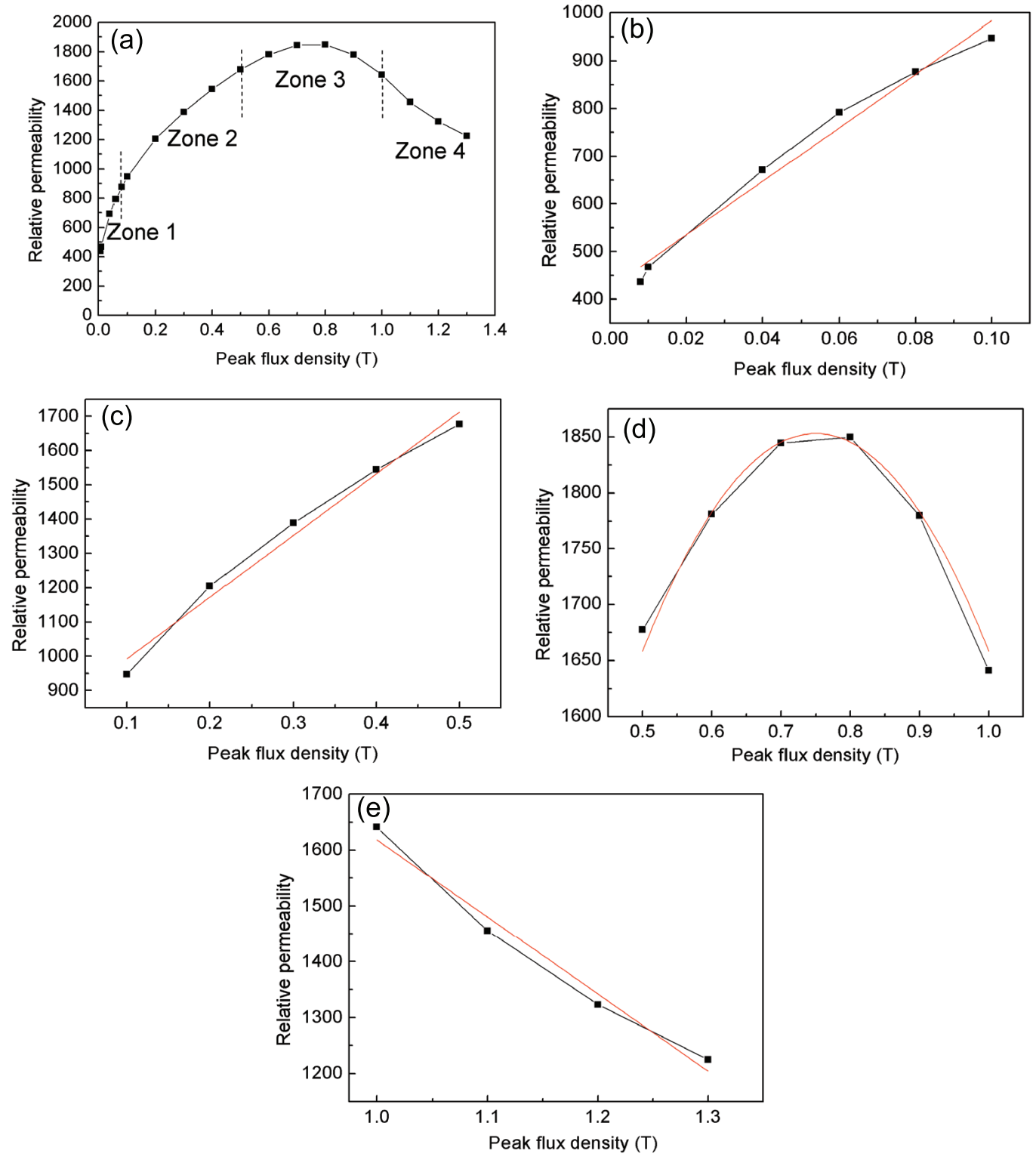

Fig. 4 Curve fitting of average relative permeability of H668 after ball scribing: (a) zones separation; (b) zone 1; (c) zone 2 ; (d) zone 3 ; (e) zone 4

\section{Conclusions}

(1) Ball scribing could effectively improve relative permeability of both CGO and HGO electrical steels. Permeabilities of CGO and HGO electrical steels were increased by $109 \%$ and $80 \%$, respectively, with scribing width of $16 \mathrm{~mm}$ at $1.0 \mathrm{~T}$. The relative permeability increases as the scribing space gradually increases, during which the peak relative permeability points moves towards high flux densities.

(2) Relative permeability variations of both CGO and HGO electrical steels appear two stages, in the first stage they show identical average values, and in the second stage there is a quite similar relative permeability gap between them.

(3) Models between relative permeability and flux density were established at four different magnetiz- ing stages, which show good fitting effect. Variation of the experimental curves was analyzed in relative permeability development during the magnetization process.

\section{Acknowledgements}

This work was financial supported by National Natural Science Foundation of China (Nos. 51174057 and 51274062) and the National High Technology Research and Development Program (No. 2012AA03A503). Authors would also like to thank Wolfson Centre for Magnetics, Cardiff University for the technological support.

\section{REFERENCES}

[1] C.S. Li, H. Yang, Y.F. Wang and Y.M. Yu, J. Iron 
Steel Res. Int. 17 (2010) 46.

[2] P. Beckley, Electrical Steels for Rotating Machines, Bell \&Bain Ltd, Glasgow, 2009, pp. 19-30.

[3] M.Q. Yan, P. Yang, Q.W. Jiang, Y.J. Fu and W.M. Mao, Acta Metall. Sin. 47 (2011) 25. (in Chinese)

[4] Y.L. Yang, C.S. Liu, F.J. Sun and D. Zhang, Acta Metall. Sin. (Eng. Lett.) 18 (2005) 533.

[5] C.S. Li, Y.M. Yu, Y.F. Wang and Y.M. Yu, Mater. Mech. Eng. 35 (2011) 1. (in Chinese)

[6] Y.H. Sha, S. Li, S.C. Zhou, Y.D. Wang, X.H. Gao, Z.D. Liang and L. Zuo, Acta Metall. Sin. 40 (2004) 445. (in Chinese)

[7] O. Bottauscio, M. Chiampi, D. Chiarabaglio and M. Zucca, J. Magn. Magn. Mater. 215-216 (2000) 130.
[8] D. Miyagi, K. Miki, M. Nakano and N. Takahashi, IEEE Trans. Magn. 46 (2010) 318.

[9] F.F. Chen and S.X. Zhou, J. Magn. Magn. Mater. 239 (2002) 595

[10] D. Snell and P. Beckley, J. Magn. Magn. Mater. 55 (1994) 167

[11] H. Wang, C.S. Li, T. Zhu, B. Cai, G. Huo and N. Mohamed, J. Mater. Sci. Technol. 29 (2013) 673.

[12] A.J. Moses, Scr. Mater. 67 (2012) 560.

[13] H. Wang, C.S. Li, G. Huo, B. Cai and N. Mohamed, J. Harbin Inst. Technol. (New Series) 20 (2013) 81.

[14] N. Chukwachekwa, A.J. Moses and P. Anderson, IEEE Trans. Magn. 48 (2012) 1393. 